\title{
Chopstick surgery: a novel technique enables use of the Da Vinci Robot to perform single-incision laparoscopic surgery
}

\author{
R. A. Joseph • N. A. Salas • C. Johnson • A. Goh • \\ S. P. Cuevas • M. A. Donovan - M. G. Kaufman • \\ B. Miles · P. R. Reardon • B. L. Bass · B. J. Dunkin
}

Received: 1 May 2009/Accepted: 26 February 2010/Published online: 24 June 2010

(C) Springer Science+Business Media, LLC 2010

Introduction Single-incision laparoscopic surgery (SILS) is limited by the coaxial arrangement of the instruments. A surgical robot with "wristed" instruments could overcome this limitation but the "arms" collide when working coaxially. This video demonstrates a new technique of "chopstick surgery," which enables use of the robotic arms through a single incision without collision.

Methods Experiments were conducted utilizing the da Vinci $S^{\circledR}$ robot (Sunnyvale, CA) in a porcine model with three laparoscopic ports $(12 \mathrm{~mm}, 2-5 \mathrm{~mm})$ introduced through a single "incision." Pilot work conducted while performing Fundamentals of Laparoscopic Surgery (FLS) tasks determined the optimal setup for SILS to be a triangular port arrangement with $2-\mathrm{cm}$ trocar distance and remote center at the abdominal wall. Using this setup, an experienced robotic surgeon performed a cholecystectomy and nephrectomy in a porcine model utilizing the "chopstick" technique. The chopstick arrangement crosses the instruments at the abdominal wall so that the right

Electronic supplementary material The online version of this article (doi:10.1007/s00464-010-1107-x) contains supplementary material, which is available to authorized users.

Presented at the SAGES 2009 Annual Meeting, April 22-24, 2009, Phoenix, AZ.

R. A. Joseph $(\bowtie) \cdot$ N. A. Salas · C. Johnson ·

S. P. Cuevas - M. A. Donovan - M. G. Kaufman - B. Miles ·

P. R. Reardon - B. L. Bass - B. J. Dunkin

Department of Surgery, Methodist Institute for Technology

Innovation and Education (MITIE ${ }^{\mathrm{TM}}$ ), The Methodist Hospital,

Houston, TX, 77030 USA

e-mail: rajoseph@tmhs.org

A. Goh

Department of Urology, Baylor College of Medicine, Houston,

TX, USA instrument is on the left side of the target and the left instrument on the right. This arrangement prevents collision of the external robotic arms. To correct for the change in handedness, the robotic console is instructed to drive the "left" instrument with the right hand effector and the "right" instrument with the left.

Results Both procedures were satisfactorily completed with no external collision of the robotic arms, in acceptable times and with no technical complications. This is consistent with results obtained in the box trainer where the chopstick configuration enabled significantly improved times in all tasks and decreased number of errors and eliminated instrument collisions.

Conclusion Chopstick surgery significantly enhances the functionality of the surgical robot when working through a small single incision. This technique will enable surgeons to utilize the robot for SILS and possibly for intraluminal or transluminal surgery.

Disclosures Drs. Rohan A. Joseph, Nilson A. Salas, Alvin Goh, Patrick R. Reardon, Barbara L. Bass, and Brian J. Dunkin, and Mr. Matthew Kauffman, Mike Donovan, and Chris Johnson have no conflicts of interest or financial ties to disclose. 